contributed to the diagnosis of FLNA mutation including diffuse ground glass opacification and persistent interstitial changes on Chest Radiographs and CT as well as periventricular heterotopia on MRI Brain.

\section{P549 SHOULD CHILDREN WITH DOWN SYNDROME RECEIVE PROPHYLACTIC ANTIBIOTICS TO PREVENT RECURRENT RESPIRATORY INFECTIONS?}

${ }^{1}$ Evan Chia, ${ }^{1}$ Allison Clark, ${ }^{1}$ Breanna Flynn, ${ }^{1}$ Michael Mc Kenna, ${ }^{1}$ Nyin Nyee $\mathrm{Ng}$, ${ }^{1}$ Sophie Sherry, 'Dylan Stacey, 'Zhong Hern Tey, Dr ${ }^{2}$ John Allen*, Dr ${ }^{2}$ Niamh Lagan, Dr ${ }^{2} J u d i t h$ Meehan, ${ }^{2}$ Prof Eleanor Molloy. 'School of Medicine, Trinity College Dublin, Dublin, Ireland; ${ }^{2}$ Department of Paediatrics, Trinity College Dublin, Dublin, Ireland

\subsection{6/archdischild-2019-epa.883}

Introduction Trisomy 21, also known as Down syndrome (DS), is a clinical disorder where a third copy of chromosome 21 is present. Approximately $95 \%$ of DS are due to a meiotic nondisjunction, with the remaining 5\% caused by chromosomal translocation or mosaicism. Children with DS are predisposed to recurrent respiratory infections due to a number of anatomical and immunological features. Our project investigated whether there is evidence supporting or refuting the use of antibiotic prophylaxis for recurrent respiratory infections in the DS population.

Methods A systematic literature review was conducted of published medical literature within the following databases: MEDLINE, Science Direct, and The Cochrane Library. A systematic search for ongoing clinical trials and guidelines/consensus statements was performed using various clinical trial registers and professional organisation websites. Search terms included 'DS', 'Trisomy 21', 'paediatric', 'respiratory infections', 'recurrent respiratory infections', 'prophylaxis' and 'antibiotics'. Systematic reviews, meta-analyses, randomised controlled trials, case-control studies and case-series were considered.

Results A systematic search revealed 0 published articles and 0 clinical trials meeting the necessary inclusion criteria. 1 guideline was found meeting our inclusion criteria; the Nottingham Guideline which outlines the role of prophylactic antibiotics in the DS population. Given the dearth of evidence in this area, we formulated a clinical trial to investigate the utility of prophylactic antibiotics for current respiratory infections in the DS population. Azithromycin was chosen as the antibiotic of choice for its anti-inflammatory and immunomodulatory properties. Primary endpoints would be the number of respiratory infections experienced over the course of the treatment period requiring a GP or ED attendance. Secondary endpoints include the severity of respiratory infections, both the number and severity of adverse events experienced over the period of the trial, along with the patient and parent/legal guardian selfreported quality of life.

Conclusion There is a current lack of evidence supporting or refuting the use of prophylactic antibiotics for recurrent respiratory infections in DS. Basic scientific studies need to be performed elucidating the role of anatomical and immunological features in predisposing children with DS to recurrent respiratory infections. Clinical trials are needed to elucidate whether prophylactic antibiotics are useful in this cohort and to investigate the optimal timing and combination of antibiotics. Guidelines are needed to support physicians in clinical decision making.

\section{P550} PNEUMONIA IN CHILDREN: DIAGNOSTIC POSSIBILITIES IN UKRAINE

${ }^{1}$ Oleksandr Volosovets, 'Sergii Krywopustov, 'Viktoriia Khomenko*, 'Oksana lemets, ${ }^{2}$ Tetiana Umanets. ${ }^{1}$ O. O. Bogomolets National Medical University, Kyiv, Ukraine; ${ }^{2}$ Institute of Pediatrics, Obstetrics and Gynecology named by academician O. Lukyanova of the National Academy of Medical Sciences of Ukraine, Kyiv, Ukraine

\subsection{6/archdischild-2019-epa.884}

Background Pneumonia is the third most common fatal cause for children under age five in Ukraine. The increase in the incidence of acute respiratory infections requires the determination of the role of pathogens in the etiology of pneumonia. The spectrum of etiological agents of community-acquired pneumonia (CAP) has not been studied in Ukraine.

The aim of study

To identify and improve the understanding of the causative role of infectious pathogens in etiology childhood CAP during the epidemic season.

The methods 49 children from 2 months to 16 years old with pneumonia were examined and treated at the Eurolab clinic (Kyiv) from September to January 2018/2019. The diagnosis was based on clinical examination, chest radiography, blood test, Multiplex PCRs for throat and nasals wabs, rapid influenza diagnostic tests (RIDTs).

Results Pneumonia was confirmed with a chest x-ray in 36 patients. The diagnosis was determined without radiographic findings, based on clinical symptoms (cough, localized crackles, or decreased breath sounds, fever, tachypnea) and hypoxemia in 13 children over 5 years old. Viral pneumonia was diagnosed in 31 patients (63\%) with normal WBC count. It was characterized by hyperinflation with bilateral interstitial infiltrates and peribronchial cuffing on chest $\mathrm{x}$-ray in 23 children. PCR tests were used in 21 children to determine etiology: Metapneumoviruses (hMPV) - 6; Respiratory syncytial virus (RSV) - 3; Adenoviruses - 2; Mycoplasma pneumoniae - 2 (confirmed by seroconversion in IgG); Chlamydophila pneumonia - 6 (3 - with Ig G seroconversion); Mycoplasma pneumonia and Chlamydophila pneumonia - 1; Adenovirus and Influenza $A$ - 1. In 6 children with viral pneumonia, influenza $A$ was identified by RIDTs. Measles was the cause of pneumonia in 2 patients.

Conclusions The study suggests that viruses (primarily, $h M P V$, influenza and RSV) play a major role in childhood CAP. Future research is required to understand viral and bacterial colonization of the respiratory tract and the relevance of the detection of pathogens in the etiology of community-acquired pneumonia, which will reduce chest X-rays and optimize antibiotic therapy for pneumonia in children.

\section{P551 LONG-TERM EFFECTS IN CHILDREN WHO UNDERWENT RESUSCITATION IN NEONATAL PERIOD}

${ }^{1}$ Yuliya Klukhina, ${ }^{2}$ Mariya Kuropatenko, 'Lyudmila Zhelenina*. 'St. Petersburg State Pediatric Medical University, Ministry of Healthcare of the Russian Federation, Saint Petersburg, Russian Federation; 'FSBSI 'Institute of Experimental Medicine', Saint Petersburg, Russian Federation

\subsection{6/archdischild-2019-epa.885}

Background Despite significant progress in nursing premature babies, neonatal pneumonia, along with artificial lung ventilation (ALV), is a significant factor in formation of chronic nonspecific lung diseases later in life. 
The study aims to compare long-term effects of artificial lung ventilation sustained in the neonatal period in dependence on newborn babies` gestational age and the mechanical parameters of ventilation.

Materials 127 patients aged 8-11 (84 full-term babies, 43 premature babies) who were treated in the intensive care unit of St. Petersburg Children`s Hospital No. 1 in the neonatal period, underwent follow-up examination of respiratory system. Of them, 27 children were born with the gestational age of $30-34$ weeks, 16 of $35-36$ weeks, and 84 at the gestational age of $37-42$ weeks. The control group consisted of 43 children with uncomplicated neonatal period.

Results In the neonatal period, pathology of the respiratory system was detected much more often in the premature infants $(56 \%$ and $88 \% ; p<0.001)$. They developed 1 st type respiratory distress syndrome $(45 \%)$ more often than the fullterm infants, while the amniotic fluid aspiration syndrome was detected in the full-term newborns more often than in the premature ones (36\%). Transient tachypnea of the newborn was significantly more frequent in the group of full-term infants $(28 \%$ and $8 \% ; \mathrm{p}=0,026)$. Among infants with bronchopulmonary pathology, pneumonia developed in preterm babies more often $(32 \%$ and $60 \% ; \mathrm{p}=0.009)$. According to a follow-up survey, the incidence of pulmonary healthy children was comparable in the group of full-term children $(74 \%)$ and in the control group $(84 \%)$ at school age, while prematurely born children developed no bronchopulmonary pathology less often $(63 \%, p=0.05)$. Besides, prematurely born children with recurrent bronchitis developed neonatal pneumonia reliably more often $(\mathrm{p}<0.05)$. All prematurely born children who developed recurrent bronchitis or chronic nonspecific lung diseases at school age (100\%) had been on ALV with 'hard' settings in the neonatal period, whereas the same indicator among full-term infants was twice lower (50\%). Family history analysis showed that family history of bronchopulmonary diseases accompanied bronchial asthma in most cases, both in full-term (67\%) and premature children (63\%).

Conclusions Neonatal pneumonia, along with iatrogenic effects of resuscitation, is the dominant factor in formation of chronic nonspecific pulmonary diseases in catamnesis. Children treated in ICU in the early neonatal period should be considered a high-risk group for development of bronchopulmonary system pathology later in life, and they must be carefully supervised by pediatrician and pulmonologist.

\section{P552 BRITISH THORACIC SOCIETY (BTS) PNEUMONIA AUDIT: COMPLETING THE LOOP}

Ehtasham Yousaf*, M Shahid Yousuf, Catalin Soroiu, Mohammad Tariq. Midlands Regional Hospital, Portlaoise, Ireland

\subsection{6/archdischild-2019-epa.886}

Aims Our aim was to collect data on children admitted to Midlands Regional Hospital, Portlaoise (MRHP) with a diagnosis of Community Acquired Pneumonia (CAP). We looked into presenting complaints, clinical signs, investigation, management and follow-up plan made on these patients. In winter 2016/17 this data was compared with data from 16 other hospitals across the UK and Ireland as part of the British Thoracic Society's (BTS) audit on CAP. Now, in winter 2018/
19, we did re-audit to look at progress of our own department in 2 years.

Methods A retrospective chart review was carried out to identify patients with CAP. Inclusion criteria included children presenting to MRHP aged 1-15 years with a diagnosis of CAP over Oct Dec 2018.

Results There was a massive improvement regarding choice of antibiotics to treat CAP and we switched to amoxicillin in majority of cases, which is in line with recommendation of BTS, UK. Our data is comparable to international data and previous local data with regard to demographics and severity of pneumonia requiring $\mathrm{O} 2$ therapy. We still are performing more blood tests (including acute phase reactants and microbiological investigations) and chest $\mathrm{x}$-rays than other centers but there is decrease in these numbers as compared to our own previous data.

Conclusions The over diagnosis, over investigation and over treatment of CAP is leading to wasted resources and is not in adherence with best practice. After the recommendations from our first audit, there was big improvement in few domains and we are progressing towards best practice in others. We are also in the process of completion of our local guidelines and after their introduction; we expect our efficacy to improve even more.

\section{REFERENCES}

1. BTS UK community acquired pneumonia in children guidelines: https://www.britthoracic.org.uk/document-library/audit-and-quality-improvement/audit-reports/btspaediatric-community-acquired-pneumonia-audit-report-201617/https://www.britthoracic.org.uk/document-library/clinical-information/pneumonia/paediatric-pneumonia/bts-guidelines-for-the-management-of-community-acquired-pneumonia-in-children-update-2011/

\section{P553 IMPACT OF THE INTERNATIONAL GUIDELINES ON THE THERAPY OF BRONCHIOLITIS: SINGLE-CENTRE RETROSPECTIVE ANALYSIS}

Veronika Csuzdi*, Gábor Uhereczky, Éva Gács, G Ágnes Tóth, Gabriella Stéger, László Szabó. Heim Pál National Paediatric Institute, Budapest, Hungary

\subsection{6/archdischild-2019-epa.887}

The AAP bronchiolitis guidelines published in 2006 and updated in 2014 recommended supportive care with limited diagnostic testing and treatment. To comply with the international guidelines in 2014 we've started a campaign to improve the therapeutic practice of bronchiolitis at our hospital.

Aim To assess the effect of the therapeutic regime changes on the morbidity of bronchiolitis (need of PICU transmission, length of hospital stay) at our hospital.

Methods The data of 225 patients, aged 1-12 months, hospitalized in the period of April to October between 2013 and 2018 with the diagnosis of bronchiolitis at our Pulmonology Unit was analysed retrospectively.

Results Antibiotic treatment application decreased since 2013 in each year (In 80\%-36\%-27\%-12\%-2\% of the patients respectively). Same as the antibiotic use, the systemic corticosteroid and inhalative B2 agonist administration decreased significantly $(60 \%-28 \%-17 \%-2 \%-0 \%$ and $80 \%-39 \%$ $-40 \%-13 \%-14 \%$ of the cases). Use of $3 \%$ saline inhalation increased: $30 \%-39 \%-93 \%-93 \%-95 \%$. Although the use of drug therapies decreased during the observational period, it did not cause an increase either in the length of hospital stay (median days $6.4-6.3-6.9-4.5-5.0$ ) or in 